\title{
Parodontitis macht Herzinfarkte wahrscheinlicher
}

\section{Eine große schwedische Studie liefert neue Evidenz für den Zusammenhang zwischen der Zahngesundheit und dem kardiovaskulären Risiko.}

$\mathrm{D}$ ie Parodontitis ist eine bakterielle Entzündung, die durch einen mikrobiellen Belag auf der Zahnoberfläche hervorgerufen wird. Die Bakterien befinden sich im fortgeschrittenen Stadium der Erkrankung unterhalb des Zahnfleischrandes und können nur durch einen antiinfektiösen Eingriff entfernt werden. Unbehandelt kann die Erkrankung zu Zahnverlust führen.

In die große schwedische Fallkontrollstudie PAROKRANK wurden 805 Patienten unter 75 Jahren aufgenommen, die einen ersten Herzinfarkt in 17 schwedischen Krankenhäusern zwischen 2010 und 2014 diagnostiziert und behandelt bekamen. Initial wurden alle Studienteilnehmer klinisch untersucht. Es wurden Labortests gemacht und die Patienten mussten Fragebogen (Vorgeschichte, Familienanamnese, Depressionen) ausfüllen. Alle Patienten wurden auch zahnärztlich untersucht und bekamen eine Panoramaaufnahme der Zähne. Die Patienten waren im Mittel 62 Jahre alt, $81 \%$ waren männlich.

Zu Studienbeginn hatten mehr Patienten mit Herzinfarkt als Kontrollen einen manifesten Diabetes mellitus (19 vs. $13 \%$, $\mathrm{p}<0,002)$. In der Herzinfarktgruppe waren mehr Raucher bzw. frühere Raucher (62 vs. $57 \%$ ). Auch der soziale Status war unterschiedlich (66 vs. $62 \%$ ).

Es zeigte sich, dass Patienten mit einem ersten Herzinfarkt in 43 versus $33 \%$ (Kontrollen) eine Parodontitis aufwiesen. Nach der Adjustierung aller begleitenden Risikofaktoren hatten Patienten mit einer manifesten Parodontitis ein deutlich höheres Herzinfarktrisiko (Odds Ratio 1,2). Rauchen und Diabetes waren die Hauptrisikofaktoren für Parodontitis.

Ryden $L$ et al. Periodontitis increases the risk of a first Myocardial infarction: A report from the PAROKRANK Study. Circulation. 2016;133:576-83

\section{Kommentar}

International wird diese Studie als weitere Evidenz dafür angesehen, dass die Zahngesundheit ein wichtiger protektiver Faktor für das Auftreten einer koronaren Herzkrankheit ist. Die Experten empfehlen nach dem Auftreten eines akuten Koronarsyndroms unbedingt die Abklärung des Zahn-

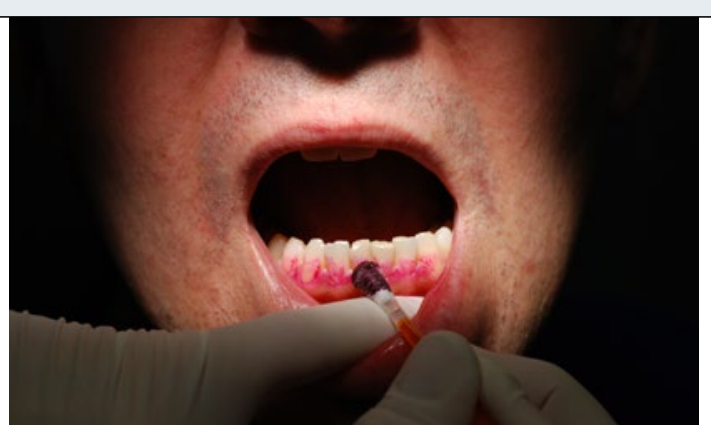

Einen Zusammenhang zwischen einer Parodontitis und dem Herzinfarktrisiko haben bereits mehrere Studien gezeigt.

status. Vice versa sollten Zahnärzte daran denken, dass sie bei Patienten mit einer ausgeprägten Parodontitis eine Überweisung zum Kardiologen veranlassen.

In den USA und wahrscheinlich auch in Europa haben Patienten über 30 Jahre in mehr als der Hälfte eine ausgeprägte Parodontitis, also eine chronische Entzündung des Zahnhalteapparates. Mehr als 50 Publikationen der letzten 25 Jahre haben Zusammenhänge zwischen chronischer Parodontitis und der Häufigkeit von Herz-Kreislauf-Erkrankungen ergeben. Die Studien waren zum großen Teil aber relativ klein und die Parodontitis war oft ungenau dokumentiert. Regelmäßiges intensives Zähneputzen und die sorgfältige Reinigung der Interdentalräume (Zahnseide oder Bürstchen) können die Entstehung einer Gingivitis/Zahnfleischentzündung verhindern. Die Gingivitis ist meist eine Vorstufe der Parodontitis.

Prof. Dr. med. Curt Diehm Max-Grundig-Klinik Bühl

\section{RAS-Blocker und Diabetes: Wackelt das Dogma?}

\section{Die Präferenz für RAS-Blocker bei Hypertonie und Diabetes mellitus ist ein seit Langem geübter und kaum infrage gestellter Brauch. Die vorliegende Metaanalyse kommt zu überraschenden Ergebnissen.}

$\mathrm{D}$ ie einschlägigen Datenbanken wurden hinsichtlich randomisierter Studien mit RAS-Blockern versus andere Antihypertensiva bei Diabetikern durchsucht. Endpunkte waren Tod, kardiovaskulärer Tod, Herzinfarkt, Angina pectoris, Schlaganfall, Herzinsuffizienz, Revaskularisierung und terminale Niereninsuffizienz. 19 randomisierte kontrollierte Studien mit 25.414 Diabetikern und 95.910 Patientenjahren wurden ausgewertet.
Für die in Abbildung 1 dargestellten Endpunkte fand sich kein signifikanter Unterschied zwischen RAS-Blockern und anderen Antihypertensiva. Es wurde eine Power von $94 \%$ für eine Reduktion der terminalen Niereninsuffizienz um $23 \%$ errechnet.

Bangalore $S$ et al. Diabetes mellitus as a compelling indication for use of renin angiotensin system blockers: systematic review and meta-analysis of randomized trials. BMJ. 2016;352:i438

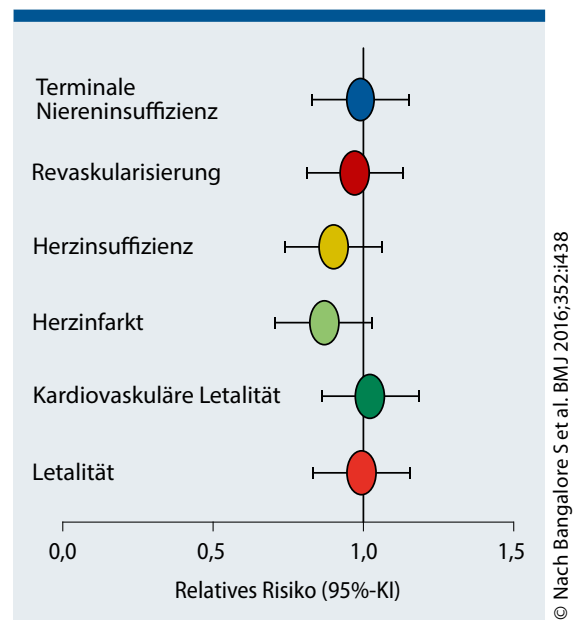

Abb. 1: Relatives Risiko verschiedener Endpunkte $(<1$ : RAS-Blocker besser, $>1$ : andere Antihypertensiva besser). 